\title{
Modelo de crecimiento arbóreo de especie Almendrón
}

\author{
Model of arboreal growth of Almendrón species.
}

\section{Espécies modelo o crescimento da árvore almendrón.}

\author{
Martínez-Lozano, José Joaquín ${ }^{1}$ \\ Zafra-Tristancho, Sandra Liliana ${ }^{2}$ \\ Vergel-Ortega, Mawency ${ }^{3}$ \\ ${ }^{1}$ Escuela Superior de Administración Pública \\ 2 Policía Nacional de Colombia- Colombia \\ ${ }^{3}$ Universidad Francisco de Paula Santander - Colombia
}

\section{Resumen}

El artículo analiza modelos de covarianza en la estimación de curvas de crecimiento arbóreo individuales y poblacionales. La investigación sigue un enfoque cuantitativo inferencial, la población incluye especies Almendrón, diez árboles por especie y una serie de ancho de anillos de crecimiento por árbol. Las series fueron suavizadas mediante medias móviles para maximizar la tendencia debida al crecimiento biológico. Resultado: modelo con efectos aleatorios asociados a cada parámetro de un polinomio de segundo orden resultó el más eficiente en la estimación del crecimiento de especies. Conclusión: efectos aleatorios permiten verificar la variabilidad del crecimiento de una especie en bosques naturales.

Palabras clave: almendrón, varianza, modelo cuadrático

\section{Abstract}

The article analyzes models of covariance in the estimation of individual and population tree growth curves. The research follows a quantitative inferential approach, the population includes species Almendrón, ten trees per species and a series of width of rings of growth per tree. The series were smoothed by moving averages to maximize the trend due to biological growth. Results: model with random effects associated to each parameter of a second order polynomial was the most efficient in estimating the growth of species. Conclusion: random effects allow to verify the variability of the growth of a species in natural forests.

Keywords: almendrón, variance, quadratic growth model

\section{Introducción}

La investigación en agricultura y forestería comúnmente presenta situaciones en las que es difícil utilizar los modelos lineales clásicos de análisis de varianza y regresión porque no se cumplen los supuestos de independencia, normalidad, igualdad de varianzas o incluso linealidad (Alpiza, 2008). La modelación de datos experimentales en el marco teórico de los modelos lineales y generalizados mixtos brinda la posibilidad de analizar datos con estructuras de dependencia, desbalances y falta de normalidad (Núñez, 2011; Pereira, 2010). No obstante, las muestras dendrocronológicas provenientes del leño de especies arbóreas permiten obtener lecturas de anchos de anillos de crecimiento para cada año de vida, lecturas obtenidas desde un mismo individuo se

1. Licenciado en Biología y Química, Especialista en Biomatemática, Magister en Gerencia Educativa. Filiación: Escuela Superior de Administración Pública. Correo correspondencia:checo.jf@gmail.com

2. Licenciada en Biología y Química, Especialista, maestría en prácticas pedagógicas. Filiación: Policía Nacional de Colombia.

3. Licenciada en Matemáticas y Física. Especialista en Estadística Aplicada. Doctora en Educación. Filiación: Universidad Francisco de Paula Santander Correo:mawency@ufps.edu.co. 
encuentran correlacionadas debido al efecto de individuo (Azen, 1972). La variable ancho de anillo de crecimiento en el tiempo $t$ contiene diferentes señales determinísticas, tales como la señal biológica (edad del árbol), climática (influencia del clima en el período de crecimiento $\mathrm{t}$ ), o debidas a disturbios del ecosistema. Esta modelación en el marco de los modelos mixtos maneja estas correlaciones mediante la incorporación de variables aleatorias o mediante la modelación directa de la matriz de covarianzas residual.

El filtrado de la serie tendiente a maximizar la señal de interés representa una práctica estadística común en el tratamiento de series de anchos de anillos de crecimiento (Yohannes, Nikolaus y Pearson, 2013). Las series de anchos de anillos suavizadas muestran también correlaciones debidas al proceso de filtrado en sí mismo. Para especies arbóreas de lento crecimiento, es frecuente observar que en el período de tiempo examinado, edad a partir de los que se obtienen las muestras dendrocronológicas, la señal debida al crecimiento biológico se puede modelar simplemente por un polinomio de bajo orden (Giménez, 1998). Cualquiera sea el modelo usado para describir la señal de crecimiento, las correlaciones entre las lecturas de ancho de anillos deben ser contempladas en la modelación.

En este trabajo, se modelan series provenientes de especies arbóreas de lento crecimiento en espacios urbanos de la ciudad de Cúcuta. Las series se suavizan para maximizar la señal de crecimiento biológico, al asumir modelos polinómicos cuadráticos para la tendencia biológica y distribución normal multivariada para componentes aleatorias del modelo. La atención se centra en diversos modelos para la covarianza entre las lecturas de ancho de anillo de crecimiento obtenidas desde un mismo individuo (Tunjano y Calvo, 2011), a partir de matriz de varianza y covarianza de términos de error del modelo polinomial de regresión y con la incorporación de efectos aleatorios en el modelo polinomial de regresión.

Los efectos de los parámetros del modelo de crecimiento, coeficientes polinomiales, se consideran fijos en el primer caso; los errores estándar y contrastes de hipótesis contemplan la covarianza entre los términos de error asociados a observaciones realizadas sobre un mismo individuo. La información proveniente de distintos individuos se asume no correlacionada. En el segundo caso, los efectos de los parámetros del modelo de crecimiento se consideran aleatorios (Graybill, 1976; Litell et al., 1996). Ambas aproximaciones se presentan en un marco unificado según el modelo lineal general.

\section{Metodología}

La investigación se enmarca dentro del enfoque mixto, desde métodos cuantitativos correlacionales (Contreras, 2012; Codina, 2013). Se analizaron 10 series de anchos de anillos de crecimiento obtenidas de muestras del leño de Caryocar amygdaliferum Mutis (Almendrón) con hojas perennes, compuestas, coriáceas, pecioladas, ternadas o bipinnadas (5 hojas), flores hermafroditas agrupadas en inflorescencias 0 racimos terminales, fruto drupa, semilla oleosa y comestible, cuyo aceite llamado "de piquia" se usa para freir; especie características del espacio urbano de la ciudad de Cúcuta y regiones tropicales y subtropicales, caracterizada por ubicación en borde de bosque (Vanegas, 2013; Ramos, Mayo, Tovar, 2012). Las variaciones de diámetro de tronco fueron medidas con sensores de desplazamiento variable y de respuesta lineal tipo LVDT (Linear Variable Differential Transformer), el cual consta de núcleo magnético con bobina primaria a la cual se le aplica una señal portadora (corriente alterna $10 \mathrm{~V}$ ) que produce un campo magnético alrededor del núcleo, y este campo magnético induce un voltaje en la bobina secundaria, proporcional al desplazamiento experimentado por la especie vegetal en la cual esté instalado, con un rango de medida lineal de 5 $\mathrm{mm}$ y 1 a 10 micras de precisión. Sensores conectados a 4 dataloggers Microlsis, conectados entre sí mediante comunicación RS-485, registrando datos cada 10 minutos y recogidos vía módem. Las longitudes promedios de dichas series fueron de 12 a $15 \mathrm{~m}$ de altura, flor amarilla, 70 y 80 años para Acacia y Almendrón respectivamente. El 
crecimiento vegetativo se evaluó inicialmente en base a distribución radical, longitud de brotes, diámetro de tronco, superficie sombreada, área foliar. Las series de lecturas de anchos de anillos de crecimiento (incrementos radiales) fueron suavizadas para eliminar las variaciones de alta frecuencia, las cuales se atribuyen al clima y/o a disturbios del bosque y maximizar la tendencia biológica o aquella debida a la edad del árbol, objetivo de la modelación. Se suaviza y eliminan árboles por presentar patrón apartado con respecto al esperado para la tendencia biológica de crecimiento (Gimenez, 1999). Se realizó poda, recolección y peso de hojas, y, a partir de la relación obtenida entre el peso fresco y seco total se estimó el peso seco de las ramas de poda para el resto del período experimental.

El diámetro del tronco se analizó antes del inicio y final de la actividad del año, a partir de medidas del perímetro de tronco (Kaweesa, et. al., 2013), de árboles testigo, a una altura sobre el suelo de 40 $\mathrm{cm}$, el sistema radicular se encontró en una banda de $120 \mathrm{~cm}$ de ancho con incremento anual de $15 \%$, se encontró una elevada concentración de raíces de diámetro inferior a $1 \mathrm{~mm}$. A partir de estos datos se obtuvo el área de la sección transversal del tronco La forma general de la ecuación de un modelo mixto,

$$
Y=X \cdot+Z u+e
$$

"donde $Y$ es un vector $n \times 1$ de variables aleatorias observables (datos), X y Z matrices de diseño conocidas, - es un vector px1 de efectos paramétricos asumiendo valores fijos (efectos fijos), $u$ es un vector $m \times 1$ de variables aleatorias (efectos aleatorios) y e es un vector $n \times 1$ de términos de error aleatorios. Los vectores $u$ y e se suponen con distribución normal multivariada con esperanza cero y matriz de covarianza $G$ (de orden $\mathrm{m} \cdot \mathrm{m}$ ) y $\mathrm{R}$ (de orden $\mathrm{n} \cdot \mathrm{n}$ ) respectivamente. Además se asume que $\operatorname{Cov}(\mathrm{u}, \mathrm{e})=0$ " (Torrecillas et al.,1988). Así se tiene que $V(Y)=Z G Z+R$. Si G y $R$ son conocidas, $V$ es conocida, las soluciones acorde a método de mínimos cuadrados generalizados para estimar - representan el estimador de parámetros BLUE (Best Linear Unbiased Estimator) fijos del modelo y la solución es un BLUP (Best Linear Unbiased Predictor) de los efectos aleatorios
(Searle et al., 1992), V desconocida por lo que en una primera instancia se estiman las componentes de varianza en $G$ y $R$, obteniéndose para estimar • y u. Bajo normalidad, la estimación de los componentes de varianza y covarianza puede realizarse por procedimientos basados en la función de máxima verosimilitud (Harville, 1977). La función de máxima verosimilitud restringida (REML) (Patterson y Thompson, 1971) es usualmente preferida para obtener estimadores de componentes de varianza en el marco de un modelo mixto (Khuri et al., 1998).

Para seleccionar uno de varios modelos mixtos factibles se utilizó la prueba del cociente de máxima verosimilitud si los modelos son anidados (Litell et al., 1996), restringida como procedimiento de estimación, evaluación del logaritmo de la máxima verosimilitud restringida (LLR) para el modelo reducido y para el modelo completo. El criterio de la prueba del cociente de máxima verosimilitud fue,

$$
\mathrm{L}=\cdot 2\{\mathrm{LLR} \text { (modelo reducido) } \cdot \mathrm{LLR} \text { (modelo completo) }\}
$$

Bajo la hipótesis que establece que ambos modelos no presentan diferencias significativas en el ajuste de los datos, el estadístico distribuido como una $\cdot 2$ con grados de libertad igual a la diferencia en el número de parámetros a estimar entre ambos modelos. Si la porción correspondiente a efectos fijos de los dos modelos mixtos bajo comparación es la misma entonces esta prueba contrasta las estructuras de covarianza de ambos modelos.

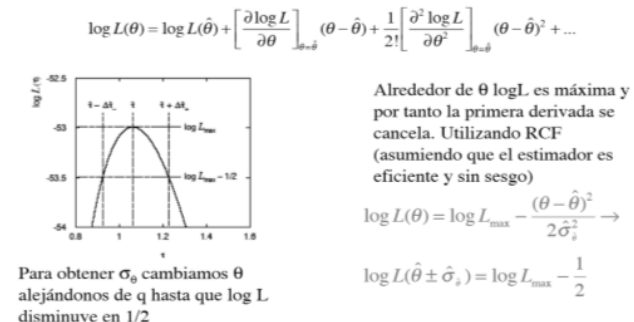

Para calcular el criterio de Akaike, se partir de la expresión:

$$
\begin{gathered}
\frac{\partial^{2} \ln (L(p))}{\partial p^{2}}=-\frac{k}{p^{2}}-\frac{n-k}{(1-p)^{2}} \leq 0 \quad \text { ya que } k \leq n \\
\text { AIC }=\log (\text { LLR)-d, }
\end{gathered}
$$


donde $\log (\mathrm{LLR})$ es el logaritmo de la función de máxima verosimilitud restringida y $\mathrm{d}$ es el número de parámetros en el modelo; valores mayores del índice AIC implican un mejor ajuste. Modelación de la covarianza entre observaciones de un mismo árbol a través de la matriz de covarianza de los términos de error (Romero, 2004). el modelo polinomial de segundo orden para tendencia biológica de crecimiento constituye la porción relacionada a efectos fijos de un modelo mixto, donde no se introduce ningún efecto aleatorio en u. y sea $\cdot i$ a la matriz de varianza-covarianza de observaciones proveniente del i-ésimo individuo y asumiendo igual estructura de covarianza en todos los individuos, si $Y$ denota el vector obtenido al apilar las series de anchos de anillos de $\mathrm{n}$ árboles una debajo de otra, la varianza de $Y$, denotada por $\mathrm{V}$, es $\mathrm{V}=\mathrm{In} \cdot \mathrm{Ri}$, donde $\cdot$ denota producto Kronecker, $\cdot \vec{i}=\mathrm{Ri}$ considerando que no hay en el modelo otro efecto aleatorio distinto de aquellos en el vector de términos de error e. Las estructuras de covarianza propuestas para modelar Ri fueron: modelo de independencia 0 modelo de componentes de varianza [CV]; modelo de simetría compuesta [SC], modelo autorregresivo de primer orden [AR(1)](Jenrich, 1986). Se asume un modelo polinomial de segundo orden para modelar el crecimiento, en este caso, en función de la edad del árbol (Girona, 1993; Guerreiro, 1998). Este crecimiento será explicado por una porción correspondiente a efectos fijos (modelo poblacional o valor esperado de la tendencia biológica de crecimiento) más un conjunto de efectos aleatorios asociados a las lecturas obtenidas desde cada árbol que conformarán el vector u del modelo mixto (Graybill, 1976). El número de efectos aleatorios adicionados en $u$ fue uno por individuo o uno por cada parámetro a ser estimado en la ecuación polinomial de crecimiento de cada árbol. Dada •i la matriz de varianzacovarianza de las observaciones proveniente del iésimo árbol y asumiendo igual estructura de covarianza en todos los individuos, $Y$, el vector obtenido al apilar las series de anchos de anillos de $n$ árboles una debajo de otra, la varianza de $Y$, denotada por $\mathrm{V}$, es $\mathrm{V}=\operatorname{Diag}(\cdot \mathrm{i})$, matriz particionada diagonal, con $\cdot i=Z_{i} G_{i} Z_{i^{\prime}}+R_{i}$ con $i=1, \ldots, n$ y $R_{i}=\cdot 2 l_{a}$. El blue de los efectos fijos contenidos en $\cdot$ en combinación lineal con los BLUPs de efectos aleatorios en u permiten formular un modelo de crecimiento diferente para cada individuo (Zeger et al., 1988).

Cuando sólo un término aleatorio, representando el efecto árbol, es introducido en el modelo, la estructura de $G_{i}$ sigue el modelo de componentes de varianza, i.e. $G_{i}=I_{a}$ donde $I_{a}$ es la matriz identidad de orden a, con a igual a la longitud de la serie de anchos de anillos para el árbol i. estrategia de modelación sujeto específico[MSE]. Al modelar adicionando un término aleatorio para cada uno de los coeficientes polinómicos, la ecuación del modelo se re-escribe como,

$$
y_{i j}=\left(\cdot 0+u_{i 0}\right)+\left(\cdot 1+u_{i 1}\right) t+\ldots .+\left(\cdot q+u_{i q}\right) t_{q}+e_{i j}(4)
$$

donde los coeficientes $\cdot 0, \ldots, \cdot q$ son constantes desconocidas estimadas $\mathrm{y} \mathrm{u}_{\mathrm{i}}$ es una variable no observable que representa una desviación aleatoria de la ordenada al origen de la curva de crecimiento del sujeto i-ésimo respecto $\cdot 0$, $\mathrm{ui}_{1}$ es otra variable que representa la desviación aleatoria del coeficiente lineal de la función de crecimiento especie i-ésima respecto $a \cdot 1$ hasta uiq. La matriz $G$ del modelo mixto correspondiente es $G$ $=\operatorname{Diag}\left(G_{1}, \ldots, G_{n}\right) \operatorname{con} G i=I_{3}$.

\section{Resultados}

Las diferencias de diámetro de tronco no fueron significativamente diferentes. $\mathrm{El}$ tratamiento mostró tasas de crecimiento diario prácticamente constantes, con un valor medio de $0.2 \mathrm{~mm} \mathrm{día}^{-1}$ en todo el periodo de crecimiento (abril 2012- dic 2013), tasas disminuyeron, alcanzando valores de $0.125 \mathrm{~mm}$ día $^{-1}$ en 2014.

Datos obtenidos a partir de los sensores LVDT se muestra la media de las fluctuaciones del diámetro de tronco por mes, caracterizado por un período de 6 días (Zeger, 1988). Se observa como la actividad del cambium comienza a finales de segundo mes, coincidiendo con una máxima floración y la emergencia de nuevos brotes y hojas. La expansión cambial continúa hasta finales de octubre, con un crecimiento relativamente 
Revista LOGOS CIENCIA \& TECNOLOGÍA

ISSN 2145-549X | ISSN 2422-4200, Vol. 5, No. 2, Enero - Junio 2014

constante hasta últimos de agosto, reducción gradual en septiembre y mínimo crecimiento en octubre, coincidiendo con los primeros síntomas de senescencia de las hojas El incremento anual del diámetro de tronco (ADT $=-0.14 \mathrm{~S}_{\text {rtallo }}+0.95$, $R^{2}=0.94$ en 2012; $A D T=-0.01 S_{\text {rtallo }}+19.4, R^{2}=0.79$ ) correlacionó negativamente con la integral de estrés y mostró $R^{2}$ altos. Las pendientes de las rectas indican el alto nivel de sensibilidad del crecimiento del tronco al aumento de la integral de estrés. La superficie sombreada al final del periodo experimental alcanzó el $35 \% \mathrm{El}$ área foliar (AF) de los árboles pasó de 6 a $30 \mathrm{~m}^{2}$; no se observaron diferencias de tamaño de hoja entre individuos; el incremento de diámetro de tronco frente a superficie del tallo, mostró un coeficiente de determinación superior denotándose una mayor sensibilidad de éste al déficit hídrico (observaciones medio día); La superficie sombreada por el cultivo, a lo largo del período experimental, estuvo bien correlacionada con el índice de área foliar $r=0.91$.

El crecimiento total del tronco fue mayor en individuos que recibieron mayor riego (aunque no fue una variable totalmente controlada en el estudio), se observa que el potencial del tallo es sensible a estado de hidratación del suelo, es decir a la hidratación hídrica. Los valores medios del incremento anual del diámetro de tronco en base a los sensores LVDT fueron de $43.5 \mathrm{~mm}$. Ahora bien, teniendo en cuenta diámetro para estimar modelo siendo en Tabla $1,-2$ Res $\log L=-2$ veces el logaritmo de la función de máxima verosimilitud restringida; AIC: criterio de Información de Akaike; MAE: media del valor absoluto de los residuos para un nivel $\alpha=0.01$, todos los contrastes de hipótesis fueron significativos

Tabla 1. Variable respuesta: lectura de anchos de anillos de crecimiento en individuos

\begin{tabular}{cccccc}
\hline Criterio $^{1}$ & CV & SC & AR(1) & MSE & MCA \\
\hline-2 Res Log L & -1601 & -1038 & -1943 & -1028 & -1140 \\
& & $\chi_{1}^{2}=135.6$ & $\chi_{1}^{2}=1031$ & & \\
\hline AIC & 400 & 1017 & 1469 & 1017 & 1071 \\
\hline MAE & 0.017 & 0.017 & 0.018 & 0.010 & 0.015 \\
\hline
\end{tabular}

Tabla 2. Estimación de parámetros del modelo

\begin{tabular}{|c|c|c|c|c|}
\hline Parámetro & SC & $\mathbf{A R}(\mathbf{1})$ & MSE & MCA \\
\hline $\begin{array}{l}\text { Ordenada al } \\
\text { origen }\left(\beta_{0}\right)\end{array}$ & $\begin{array}{l}0.2158 \\
(0.0050)\end{array}$ & $\begin{array}{l}0.1221 \\
(0.0158)\end{array}$ & $\begin{array}{l}0.1388 \\
(0.0105)\end{array}$ & $\begin{array}{l}0.1378 \\
(0.0073)\end{array}$ \\
\hline $\begin{array}{l}\text { Pendiente } \\
\left(\beta_{1}\right)\end{array}$ & $\begin{array}{l}0.0040 \\
(0.0003)\end{array}$ & $\begin{array}{l}0.0052 \\
(0.0008)\end{array}$ & $\begin{array}{l}0.0039 \\
(0.0002)\end{array}$ & $\begin{array}{l}0.0043 \\
(0.0006)\end{array}$ \\
\hline $\begin{array}{l}\text { Coeficiente } \\
\text { Cuadrático } \\
\left(\beta_{2}\right)\end{array}$ & $\begin{array}{l}-0.000031 \\
(0.000004)\end{array}$ & $\begin{array}{l}-0.000048 \\
(0.00001)\end{array}$ & $\begin{array}{l}-0.000030 \\
(0.0000001)\end{array}$ & $\begin{array}{l}-0.000042 \\
(0.00001)\end{array}$ \\
\hline
\end{tabular}

Tabla 3. Curvas de crecimiento individuales para un modelo polinomial cuadrático con coeficientes aleatorios.

\begin{tabular}{ll}
\hline Árbol & \multicolumn{1}{c}{ Modelo } \\
\hline 1 & $y_{i j}=0.2256-0.0023 t-0.00001 \mathrm{t}^{2}$ \\
3 & $y_{i j}=0.2389+0.0003 t-0.00001 \mathrm{t}^{2}$ \\
4 & $y_{i j}=0.2220-0.0002 \mathrm{t}+0.00002 \mathrm{t}^{2}$ \\
5 & $y_{i j}=0.1945+0.0024 t-0.00002 \mathrm{t}^{2}$ \\
6 & $y_{i j}=0.1733-0.00026 \mathrm{t}+0.00001 \mathrm{t}^{2}$ \\
7 & $y_{i j}=0.1609+0.0029 \mathrm{t}-0.000002 \mathrm{t}^{2}$ \\
8 & $y_{i j}=0.1653+0.0023 \mathrm{t}-0.000001 \mathrm{t}^{2}$ \\
10 & $y_{i j}=0.2165+0.0003 \mathrm{t}+0.000001 \mathrm{t}^{2}$ \\
\hline
\end{tabular}

Los ajustes polinomiales para las series de árboles individuales bajo el modelo MCA para la estructura de covarianza, se presentan en la Tabla 3. Obtenidos al adicionar desviaciones aleatorias predichas (BLUPs) a las correspondientes estimaciones de los coeficientes de regresión poblacional (Vanclay, 1994) , yij j-ésima representa diámetro de crecimiento $\mathrm{o}$ incremento radial suavizado para el individuo $i$, $t$ edad del árbol en la que se registra el ancho de anillo j-ésimo

\section{Conclusiones}

Existe relación entre el índice de área foliar y la superficie sombreada; la tasa de crecimiento del tronco, también tiene una alta sensibilidad al estado hídrico de la planta. En condiciones de sequía, las evoluciones de las diferencias en diámetro respecto al control son muy similares, lo cual permite generar un modelo predictivo. 


\section{Revista LOGOS CIENCIA \& TECNOLOGÍA}

Artículo de investigación

ISSN 2145-549X | ISSN 2422-4200, VoL. 5, No. 2, Enero - Junio 2014

La reducción de la dimensionalidad de un modelo de curva de crecimiento se puede realizar a través de la modelación con patrones más estructurados de la matriz de varianza y covarianza de los términos de error y/o mediante la incorporación de efectos aleatorios (de individuos) en el modelo. Si bien, las curvas de crecimiento obtenidas varían de individuo a individuo, no existe diferencia significativa en condiciones de riego similar.

\section{Referencias bibliográficas}

Alpizar E.; E. Dechamp, F. Lapeyren Montes, C. Guilhaumon, B. Baertrand, C. Jourdan. et al. (2008). Agrobacterium rhizogenes-transformed roots ofCoffee (C. arabica): Conditions for longtermproliferation and morphological and molecularcharacterization. Ann Bot 101 (7). 929940.

Azen, S. P. y A. A. Afifi. (1972). Asymptotic and small-sample behavior of estimated Bayes rules for classifying time-dependent observations. Biometrics, 28, 989-998.

Codina Canet, M. A., Olmeda Gómez, C., \& Perianes Rodríguez, A. (2013). Analysis of Scientific Production and Research Specialization of the Universidad Politécnica de Valencia, Scopus (20032008), 36(3), $\quad 17 \quad$ p. https://doi.org/10.3989/redc.2013.3.942

Guerreiro O.; P. Denolf, M. Peferoen, B. Decazy, A. Eskes and R. Frutos. (1998). Susceptibility of the Coffee Leaf Miner (Perileucoptera spp) to B.thuringiensis --endotoxins: A model for transgenic perennial crops resistant to endocarpic insects. Current Microbiology 36. 175-179.

Graybill, F. A. (1976) Theory and Application of the Linear Model. Wadsworth Publishing Company. 704 pp.

Contreras Bello, Y. (2012). Los elementos de la investigación: como reconocerlos, diseñarlos y construirlos. Autor: Hugo Cerda Gutiérrez. Colombia: Editorial Magisterio, 2011, 521 pp.
Revista Logos Ciencia \& TecnologíA, 4(1), 220-221. Recuperado de http://revistalogos.policia.edu.co/index.php/rlct/art icle/view/183/194

Contreras Bello, Y. (2012). Bases de la Investigación Cualitativa, técnicas y procedimientos para desarrollar una teoría fundamentada. Autores: Anselm Strauss y Juliet Corbin. Universidad de Antioquia, Colombia, 2012. Revista Logos Ciencia \& Tecnología, 3(2), 172-173. doi:http://dx.doi.org/10.22335/rlct.v3i2.166

Giménez, A.M.; Ríos N.A.(1999). Crecimiento de Schinopsis Quebracho colorado (Schlecht.) Barkl et meyer, Anacardiaceae. Madera y Bosques 5(2). 3551.

Kaweesa, S. H., Jonkvorst, R. J., Katebaka, R., Ssemmanda, R., Pomeroy, D., \& Brouwer, J. (2013). Is the Hamerkop Scopus umbretta a neo-colonist or an opportunist nester? Scopus, 32, 35-38.

Kanno T.; S. Naito and K. Shimamoto. (2000). Posttranscriptional Gene Silencing in Cultured Rice Cells. Plant Cell Physiol 41 (3): 321-326.

Leroy T.; A. Henry, M. Royer, I. Altosaar, R. Frutos and R. Phillipe. (2000). Genetically modified coffee plants expressing the $B$. thuringiensis cry1Ac genefor resistance to leaf miner. Plant Cell Rep 19: 382-389.

Libiakova G.; B. Jorgensen, G. Palmgren, P. Ulvskor and E. Johansen. (2001). Efficacy of an introncontainingkanamicin resistance gene as a selectable marker in plant transformation. Plant Cell Rep 20: 610-615.

Littell, R. C.; G. A. Milliken; W.W. Stroup y R. D. Wolfinger. (1996). SAS System for Mixed Models. Cary, N.C.: SAS Institute Inc.633pgs.

Miranda, H. I. C., Aguiar, P. R., Euzebio, C. D. G., \& Bianchi, E. C. (2010). Fuzzy logic to predict thermal damages of ground parts. Scopus, 434-441.

Núñez-Lagos Roglá, R. (2011). La radioactividad ambiental. Revista Logos Ciencia \& Tecnología, 
Revista LOGOS CIENCIA \& TECNOLOGÍA

ISSN 2145-549X | ISSN 2422-4200, Vol. 5, No. 2, Enero - Junio 2014

2(2),

doi:http://dx.doi.org/10.22335/rlct.v2i2.82

50-61.

Pereira dos Anjos, M. (2010). El urbanismo en las ciudades latinoamericanas y la cooperación en la gestión urbana entre Brasil y Colombia. Revista Logos Ciencia \& Tecnología, 1(2), 152-156. doi:http://dx.doi.org/10.22335/rlct.v1i2.55

Ramos Moreno, A., Mayor Polanía, R., Ortiz P, N., \& Tovar Pérez, L. (2012). La diversidad en aves como factor determinante de la interacción entre ecosistemas del departamento del Huila. Revista Logos Ciencia \& Tecnología, 3(2), 45-58. doi:http://dx.doi.org/10.22335/rlct.v3i2.158

Ruiz-Sánchez, M.C., Torrecillas, A., DelAmor, F., León, A. y Abrisqueta, J.M. 1988. Leaf water potential and leaf conductance during the growingseason in almond trees under different irrigation regimes. Biologia Plantarum (30):327-332

Simonneau, T., Habib, R., Goutouly, J.P. y Huguet, J.G. 1993. Diurnal changes in stem diameter depend upon variations in water content: direct evidence in peach trees. Journal of Experimental Botany (44):615-621.

Syvertsen, J.P., Lloyd, J. y Kriedemann, P.E. 1988. Salinity and drought stress effects on foliar ion concentration, water relations, and photosynthetic characteristics of orchard citrus. Australian Journal of Agricultural Research (39):619-627.

Smallie, J., \& Virani, M. Z. (2010). A preliminary assessment of the potential risks from electrical infrastructure to large birds in Kenya. Scopus, 30, 32-39. https://doi.org/10.2193/2008-525

Tabuenca, M.C. y Herrero, J. 1966. Influencia de la temperatura en la época de floración de los frutales. An Aula Dei (8):115-153.

Tardieu, F. y Simonneau, T. 1998. Variability among species of stomatal control under fluctuating soil water status and evaporative demand: modelling isohydric and anisohydric behaviours. Journal of Experimental Botany (49):419-432.

Torrecillas, A., Domingo, R., Galego, R. y RuizSánchez, M.C. 2000. Apricot tree response to withholding irrigation at different phonological periods. Scientia Horticulturae (85):201-215.
Tunjano Huerta, C., \& Calvo Valencia, D. (2011). Evaluación de sustancias fitoprotectoras usadas como estrategia de neutralización de la acción del glifosato sobre cultivos de erythroxylum coca. Revista Logos Ciencia \& Tecnología, 2(2), 26-31. doi:http://dx.doi.org/10.22335/rlct.v2i2.79

Yohannes, E., Nikolaus, G., \& Pearson, D. J. (2013). Stable isotopes of soil collected from feet of two species of migratory Acrocephalus give clues to stopover sites. Scopus, 32, 1-9.

Vanegas, A. y Ospina A.M. Colombia: Editorial Ibáñez (2013), Revista Logos Ciencia \& Tecnología, 4(2), 168-169. Recuperado de http://revistalogos.policia.edu.co/index.php/rlct/art icle/view/200

Vélez, J.E., Intrigliolo, D.S. y Castel, J.R. 2007. Scheduling deficit irrigation of citrus trees with maximum daily trunk shrinkage. Agricultural Water Management (90):197-204.

Vonesh, E. y Chinchilli, V. (1997). Linear and nonlinear models for the analysis of repeated measurements. Statistic textbooks and Monographs, Inc. New York Ed. Marcel Dekker, 560

Wolfinger, R. D. y O'Connell, M. (1993). Generalized linear mixed models: a pseudo-likelihood approach. J. Statist. Comput. Simul. 48:233-243.

Zeger, S. L.; K. Y. Liang y P. S. Albert. (1988). Models for longitudinal data: A generalized estimating equation approach. Biometrics 44:1049-1060. 